\title{
Can patients with recurrent high-risk prostate cancer after external beam radiotherapy (EBRT) be salvaged by low dose rate (LDR) brachytherapy: case report describing technique and application
}

\author{
Keisei Okamoto ${ }^{1,2}$ \\ ${ }^{1}$ Department of Brachytherapy for Prostate Cancer, Shiga University of Medical Science, Tsukinowa, Seta, Shiga, Japan; ${ }^{2}$ Department of Urology, \\ Uji Hospital, Kyoto, Japan \\ Correspondence to: Prof. Keisei Okamoto MD, D. Med. Sci. Department of Brachytherapy for Prostate Cancer, Shiga University of Medical Science, \\ Tsukinowa, Seta, Shiga 520-2192, Japan. Email: keiseiok814@gmail.com.
}

\begin{abstract}
Management of local relapse in prostate cancer after initial treatment is a serious issue. Many salvage options exist for patients who have local recurrence after external beam radiotherapy. Low-doserate (LDR) brachytherapy (BT) is a treatment option for patients with a recurrence of prostate cancer after external beam radiotherapy (EBRT). However, the recurrence of high-risk prostate cancer usually precludes patients from salvage treatment. The author presents two successful cases by salvage LDR BT for recurrence of high risk prostate cancer after external beam EBRT. Case 1: a 61 years old man with high risk prostate cancer presenting prostate-specific antigen (PSA) of $61 \mathrm{ng} / \mathrm{mL}$, Gleason's score 4+3, and clinical T3a disease. The patient received intensity modulated radiotherapy (IMRT) total dose of 74.8 Gy in 2009. PSA reached nadir of $0.938 \mathrm{ng} / \mathrm{mL}$ in 2011. Later on PSA kept on increasing and reached $2.604 \mathrm{ng} / \mathrm{mL}$. MRI showed local recurrence in the right lobe of the prostate. Case 2: a 60 years old man with high risk prostate cancer presenting PSA of $13 \mathrm{ng} / \mathrm{mL}$, Gleason score 4+3, and clinical T3a disease. The patient received EBRT total dose of 70 Gy in 2005. Both of the patients have stayed healthy with biochemical failure free state and without urinary and bowel troubles at seven years after salvage LDR BT. Technical points to successful salvage in these difficult cases are also reported in detail. In conclusion, salvage LDR BT can be a viable treatment option even for high-risk prostate cancer recurrence after EBRT.
\end{abstract}

Keywords: Case report; salvage brachy therapy; external beam radiotherapy failure (EBRT failure); high risk prostate cancer

Received: 13 June 2021; Accepted: 23 August 2021; Published: 30 December 2021.

doi: $10.21037 /$ tro-21-12

View this article at: https://dx.doi.org/10.21037/tro-21-12

\section{Introduction}

Diagnoses of nonmetastatic prostate cancer have been rapidly increasing given the broad adoption of prostatespecific antigen (PSA) screening (1). Most of these patients select radical treatments, but those with low-risk prostate cancer prefer active surveillance. In 2011, Nguyen et al. warned that newer and costlier prostate cancer therapies were rapidly and widely adopted, causing excess national spending without thorough comparative effectiveness research (2). Ten years later, the trend continues with the advent of robotic surgery or proton beam radiotherapy.

Despite the development of new technology, local control remains elusive, with biochemical failure ultimately occurring in $40 \%$ to $60 \%$ of the patients (3). This means that half of the patients who received initial prostate cancer treatment encounter treatment failure. Most biochemical failure is caused by localized tumor recurrence, not a metastatic disease (4). Patients who have biochemical failure 
Table 1 Initial diagnosis and primary EBRT

\begin{tabular}{lll}
\hline & Case 1 & Case 2 \\
\hline Age at diagnosis (years) & 61 & 65 \\
PSA before treatment $(\mathrm{ng} / \mathrm{mL})$ & 61.0 & 12.6 \\
Gleason score at initial biopsy (ratio of positive cores/total sampling cores) & $4+3(7 / 8)$ & $4+3(4 / 6)$ \\
T stage & T3a & T3a \\
NCCN risk category & High Risk & High Risk \\
Primary EBRT (Gy) & IMRT (74.8 Gy) & 3D CRT (70 Gy) \\
Neoadjuvant/adjuvant ADT for primary EBRT & Not conducted & Six months before and after EBRT \\
PSA nadir (ng/mL) & 0.938 & 0.100 \\
\hline
\end{tabular}

PSA, prostate-specific antigen; IMRT, intensity-modulated radiotherapy; EBRT, external beam radiotherapy; 3D CRT, three-dimensional conformal radiation therapy; ADT, androgen deprivation therapy; NCCN, National Comprehensive Cancer Network.

commonly do not receive potentially curative second-line treatment and are treated by palliative androgen depression therapy (ADT) $(3,5,6)$. Therefore, management of local relapse in prostate cancer after initial treatment is a serious issue. Many salvage options exist for patients who have local recurrence after external beam radiotherapy (EBRT), including surgery, brachytherapy, cryotherapy, and highintensity focused ultrasound (3).

Low-dose-rate (LDR) brachytherapy (BT) is a treatment option for patients who experience a recurrence of prostate cancer after EBRT. The eligibility criteria of salvage LDR BT is initial low-risk or intermediate-risk prostate cancer $(3,5,6)$. Recurrence of high-risk prostate cancer may preclude salvage treatment. The 2019 National Comprehensive Cancer Network (NCCN) guidelines define the indication of local salvage therapy as primary stage T1 and T2 (7). Therefore, the effect of salvage LDR BT for patients with a recurrence of high-risk prostate cancer after EBRT remains unknown.

We present two patient cases with successful longterm outcomes from salvage LDR BT for high-risk prostate cancer recurrence after EBRT. Technical points to successful salvage in these difficult cases are also reported in detail. I present the following case in accordance with the CARE reporting checklist (available at https://dx.doi. org/10.21037/tro-21-12).

\section{Case presentation}

All procedures performed in studies involving human participants were in accordance with the ethical standards of the institutional and/or national research committee(s) and with the Helsinki Declaration (as revised in 2013). Written informed consent was obtained from the patients for publication of this case report and accompanying images. Each patient information was de-identified in this report. A copy of the written consent is available for review by the editorial office of this journal.

\section{Case 1}

A 61-year-old man with high-risk prostate cancer presented with a Gleason score of $4+3$, clinical T3a disease, and his PSA was $61 \mathrm{ng} / \mathrm{mL}$. In 2009, he received primary EBRT with intensity-modulated radiotherapy of $74.8 \mathrm{~Gy}$ in 34 fractions. His lowest measured PSA after EBRT was 0.938 at 26 months after EBRT (Table 1). The patient experienced Grade 1 rectal bleeding occasionally after EBRT. In 2013, due to continuously rising PSA $(2.6 \mathrm{ng} / \mathrm{mL})$, his care team performed an extensive workup including computed tomography (CT) scan, magnetic resonance imaging (MRI), and bone scans.

His care team excluded apparent distant metastasis per these evaluations that were available at that time, although the possibility of undetected micro dissemination could not be excluded. His care team conducted a templateguided saturation mapping (TGSM) biopsy at five-year intervals after EBRT completion. The MRI identified cancer recurrence in the right lobe of his prostate (Figure 1). Based on the MRI findings, his care team conducted a template-guided saturation mapping (TGSM) biopsy at five years interval after EBRT completion, collecting 30 samples, including the seminal vesicle. They identified poorly differentiated adenocarcinoma exclusively from the 

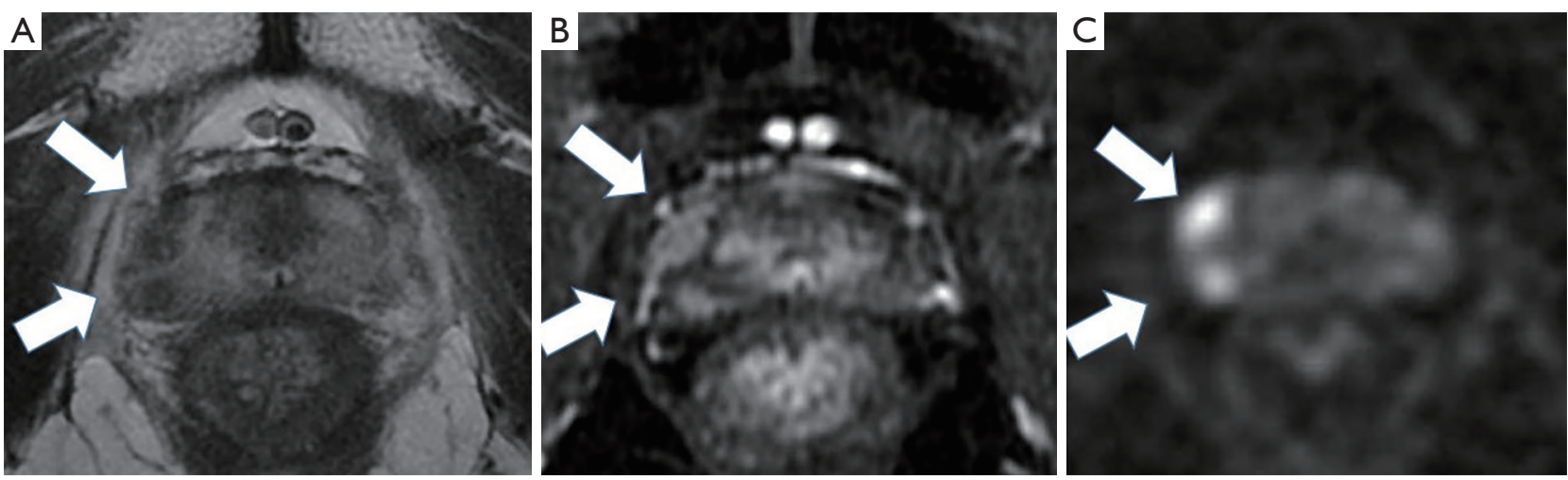

Figure 1 Axial MRI of the prostate in Case 1. (A) T2WI: white arrows indicate low-intensity recurrent prostate cancer lesion in the right lobe of the prostate. (B) Dynamic CE: white arrows indicate recurrent prostate cancer lesion with contrast enhancement in the right lobe of the prostate. (C) DWI: white arrows indicate recurrent prostate cancer lesion with a high signal area in the right lobe of the prostate. MRI, magnetic resonance imaging; T2WI, T2-weighted image; CE, contrast enhancement; DWI, diffusion-weighted image.

Table 2 Intraoperative dosimetric parameters of salvage LDR BT

\begin{tabular}{lll}
\hline Dosimetric parameter & Case 1 & Case 2 \\
\hline Prostate D90 (Gy) & 120.2 & 110.0 \\
V150 (\%) & 42.2 & 52.0 \\
V100 (\%) & 94.4 & 90.0 \\
UD30 (Gy) & 130.3 & 145.2 \\
UD5 (Gy) & 143.8 & 160.0 \\
R100 (cc) & 0.0 & 0.0
\end{tabular}

LDR, low-dose-rate; BT, brachytherapy; D90, minimal dose (Gy) received by $90 \%$ of the prostate; V150, the percentage of prostate volume receiving $150 \%$ of the prescribed minimal peripheral dose; V100, the percentage prostate volume receiving $100 \%$ of the prescribed minimal peripheral dose; UD30, minimal dose (Gy) received by $30 \%$ of the urethra; UD5, minimal dose (Gy) received by $5 \%$ of the urethra; R100, rectal volume (cc) receiving $100 \%$ of the prescribed dose.

four biopsy samples of the right lobe of the prostate, which corresponded to the MRI findings (Figure 1 and Table 2). No cancer cells were located in the remaining 26 TGSM biopsy samples where prostate tissue was replaced by fibromuscular tissue indicating an EBRT effect. Based on these findings, his care team conducted salvage LDR BT with a radiation dose of 160 Gy via ${ }^{125} \mathrm{I}$ seeds implantation to cover the MRI- and biopsy-positive lesion. The rectal volume receiving $100 \%$ of the prescribed minimal peripheral dose (R100) was 0 cc. Figure 2 presents the intraoperative dose cloud image. The dosimetric parameters of the salvage LDR BT intraoperatively and one month later are shown in Table 2 and Table 3, respectively. He experienced Grade 2 genitourinary $(\mathrm{GU})$ toxicity at four months and Grade 1 gastrointestinal (GI) toxicity eight months after salvage LDR BT.

\section{Case 2}

A 65-year-old man with high-risk prostate cancer presented with a Gleason score of $4+3$, clinical T3a disease, and his PSA was $12.6 \mathrm{ng} / \mathrm{mL}$. In 2005, he received primary threedimensional conformal radiation therapy EBRT of 70 Gy in 35 fractions. He also received neoadjuvant (six months) and adjuvant (six months) ADT before and after the EBRT. In 2011, due to rising PSA levels $(5.8 \mathrm{ng} / \mathrm{mL})$, an extensive workup was done via CT scan, MRI, and bone scans. His care team excluded apparent distant metastasis per these evaluations that were available at that time, although the possibility of undetected micro dissemination could not be excluded.

The MRI identified prostate cancer recurrence in the left posterior corner on the mid portion of the prostate (Figure 3). His care team conducted a TGSM biopsy based on the MRI findings at six-year intervals after EBRT completion, collecting 31 samples, including the seminal vesicle. They identified poorly differentiated adenocarcinoma from four biopsy samples from the left lower corner of the prostate, which corresponded to the MRI findings (Table 4). No cancer cells were located in the remaining TGSM biopsy samples where prostate tissue was replaced into fibromuscular tissue indicating EBRT effect.

Based on these findings, salvage LDR BT was conducted 

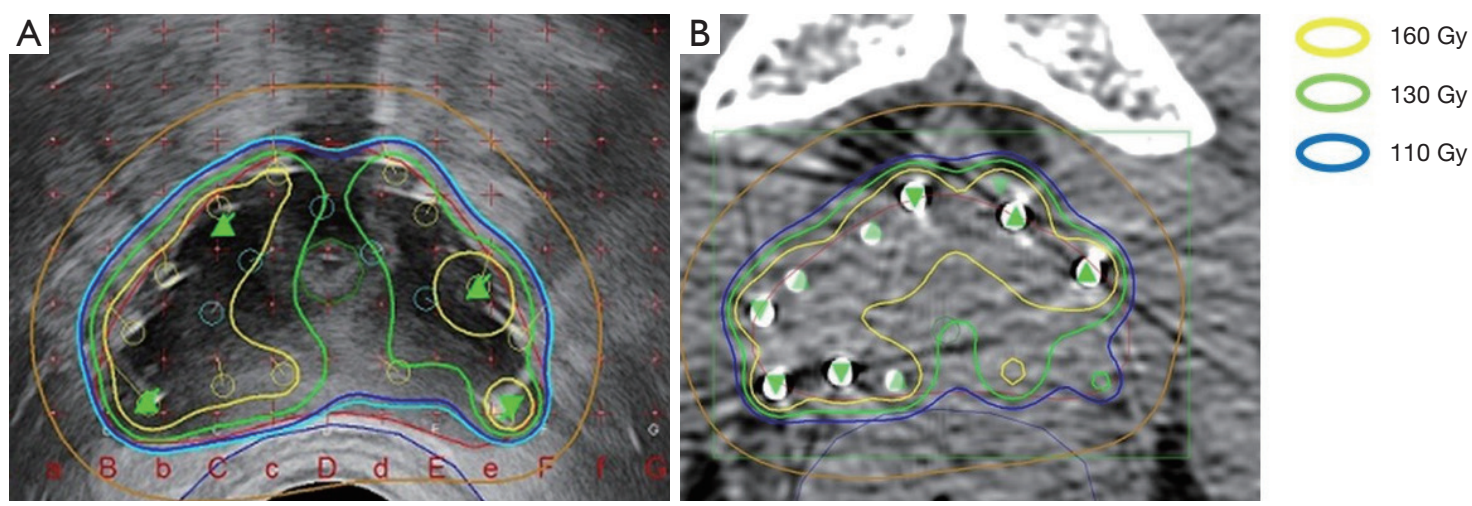

Figure 2 Dose cloud during and one month after salvage LDR BT by ${ }^{125}$ I seeds implantation in Case 1. (A) Axial dose cloud (160 Gy, yellow line) distribution during salvage LDR BT in Case $1 .{ }^{125} \mathrm{I}$ seeds implantation covers over positive lesion per MRI and TGSM biopsy (the right lobe of the prostate) and keeps rectal dose to a minimum (0 cc). The dose cloud at recurrent cancer-negative lesion proven by TGSM biopsy is designed to be limited to $130 \mathrm{~Gy}$ (green line) to $110 \mathrm{~Gy}$ (navy blue line) or lower. The red line indicates the prostate. (B) Axial dose cloud distribution one month after salvage LDR BT in Case 1. Intention of dose cloud design at the intraoperative phase is mostly achieved one month after salvage LDR BT. Red line indicates the prostate. LDR, low-dose-rate; BT, brachytherapy; MRI, magnetic resonance imaging; TGSM, template-guided saturation mapping.

Table 3 Dosimetric parameters at one month after salvage LDR BT

\begin{tabular}{lcc}
\hline Dosimetric parameter & Case 1 & Case 2 \\
\hline Prostate D90 (Gy) & 117.9 & 98.9 \\
V150 (\%) & 43.2 & 44.9 \\
V100 (\%) & 94.1 & 84.7 \\
UD30 (Gy) & 130.6 & 140.5 \\
UD5 (Gy) & 141.1 & 150.3 \\
R100 (cc) & 0.04 & 0.03 \\
\hline
\end{tabular}

LDR, low-dose-rate; BT, brachytherapy; D90, minimal dose (Gy) received by $90 \%$ of the prostate; $\mathrm{V} 150$, the percentage of prostate volume receiving $150 \%$ of the prescribed minimal peripheral dose; $\mathrm{V} 100$, the percentage prostate volume receiving $100 \%$ of the prescribed minimal peripheral dose; UD30, minimal dose (Gy) received by $30 \%$ of the urethra; UD5, minimal dose (Gy) received by $5 \%$ of the urethra; R100, rectal volume (cc) receiving $100 \%$ of the prescribed dose.

per the same protocol followed for Case 1. Figure 4 presents the intraoperative dose cloud. The dosimetric parameters of the salvage LDR BT intraoperatively and one month after salvage LDR BT are shown in Tables 2,3. He experienced no GI or GU toxicity after salvage LDR BT.

\section{Outcomes for both cases}

Both patients received neoadjuvant $\mathrm{ADT}$ two months before and adjuvant ADT 12 months after salvage LDR BT (Table 4). ADT consisted of gonadotropin-releasing hormone agonist injection and antiandrogen for each case for one year after salvage LDR BT. Outcomes were remarkably similar for both patients. Both patient's PSA levels temporarily increased after cessation of ADT, followed by continual reductions in PSA later. As of their last follow-up evaluations (each at seven years after salvage LDR BT), Case 1 had a PSA of $0.003 \mathrm{ng} / \mathrm{mL}$, and Case 2 had a PSA of $0.019 \mathrm{ng} / \mathrm{mL}$. Both patients have recovered and remained healthy without biochemical failure and without bowel and urinary troubles seven years after completing salvage LDR BT.

\section{LDR BT protocol}

Both cases presented in this report were treated using the same LDR BT protocol. Salvage LDR seed implantation was conducted with ${ }^{125} \mathrm{I}$ seeds using a real-time ultrasoundguided technique. The prescription dose of seed implantation was set at 110 Gy for LDR while the author made 160 Gy dose area on the recurrent cancer positive lesion in this protocol. The rationale of this technique is because we had to achieve the following contradictive goals in the salvage LDR-BT: We should reduce toxicity of salvage LDR-BT as much as possible as the second round of radiotherapy whereas eradicating biopsy proven recurrent cancer cells. The author thought that high radiation 

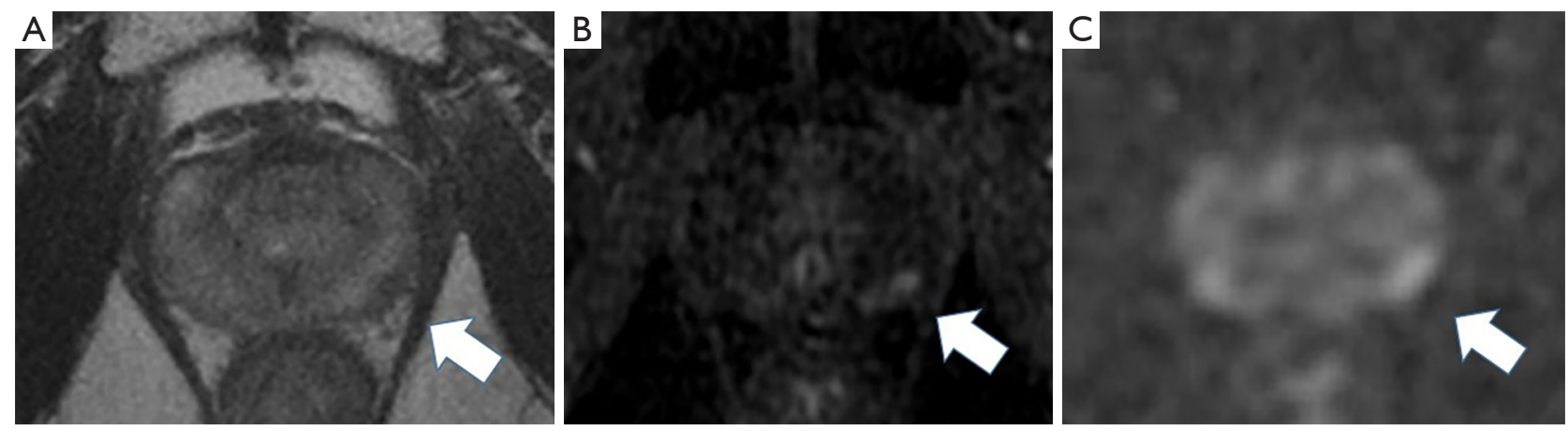

Figure 3 Axial MRI of the prostate of Case 2. (A) T2WI: white arrow indicates low-intensity recurrent prostate cancer lesion in the lower corner of the left lobe of the prostate. (B) Dynamic CE: white arrow indicates recurrent prostate cancer lesion with contrast enhancement in the lower corner of the left lobe of the prostate. (C) DWI: white arrow indicates recurrent prostate cancer lesion with high signal area recurrent prostate cancer lesion. MRI, magnetic resonance imaging; T2WI, T2-weighted image; CE, contrast enhancement; DWI, diffusion-weighted image.

Table 4 Recurrence after EBRT and outcomes of salvage LDR BT

\begin{tabular}{lll}
\hline & Case 1 & Case 2 \\
\hline Age at salvage (years) & 59 & 73 \\
PSA before salvage LDR BT (ng/mL) & 2.600 & 5.760 \\
Period to salvage LDR BT from EBRT (years) & 4.0 & 8.0 \\
Gleason score at recurrence (ratio of positive cores/total sampling cores) & Poorly differentiated & Poorly differentiated \\
& adenocarcinoma (4/30) & adenocarcinoma (4/31) \\
PSA doubling time before salvage LDR BT (months) & 5.000 & 7.000 \\
Neoadjuvant/adjuvant ADT during LDR BT & Yes & 0.019 (7 years) \\
PSA at the latest follow up (period after salvage LDR BT) & 0.003 (7 years) & 88 \\
Duration of follow up after salvage LDR BT (months) & 87 & 76 \\
Duration of follow up after cessation of ADT (months) & 75 & Biochemical failure free \\
Oncological outcome after salvage LDR BT at seven years & Biochemical failure free \\
\hline
\end{tabular}

LDR, low-dose-rate; BT, brachytherapy; PSA, prostate-specific antigen; EBRT, external beam radiotherapy; ADT, androgen deprivation therapy.

dose exposure to recurrent cancer free area only result in increased toxicity.

Radioactive seeds (seed activity at the implantation $0.284 \mathrm{mCi}$ ) were implanted into the prostate using a Mick applicator (Mick Radio-Nuclear Instruments, Ind., Mount Vernon, NY). Seeds were implanted, and the plan dosimetry was updated before continuing, so the plan evolves dynamically as seeds were implanted. Planning was performed with the VariSeed 8.0 planning system (Varian Medical Systems, CA, USA).

A dose cloud of 160 Gy by ${ }^{125} \mathrm{I}$ seeds implantation was required to eradicate recurrent prostate cancer cells on the lesion. The 160-Gy dose area must be limited to the recurrent lesion as much as possible. Radiation exposure to the rectum by LDR BT should be minimized to avoid rectal bleeding. These principles guided the LDR BT dosing procedure as follows. First, we identified the recurrent prostate cancer lesion by MRI (T2-weighted, diffusion, and dynamic contrast-enhanced). Second, we conducted a TGSM biopsy using the standard ${ }^{125} \mathrm{I}$ seeds brachytherapy template with a $5-\mathrm{mm} \times 5$-mm grid. Biopsy samples were examined by a pathologist familiar with the diagnosis of 

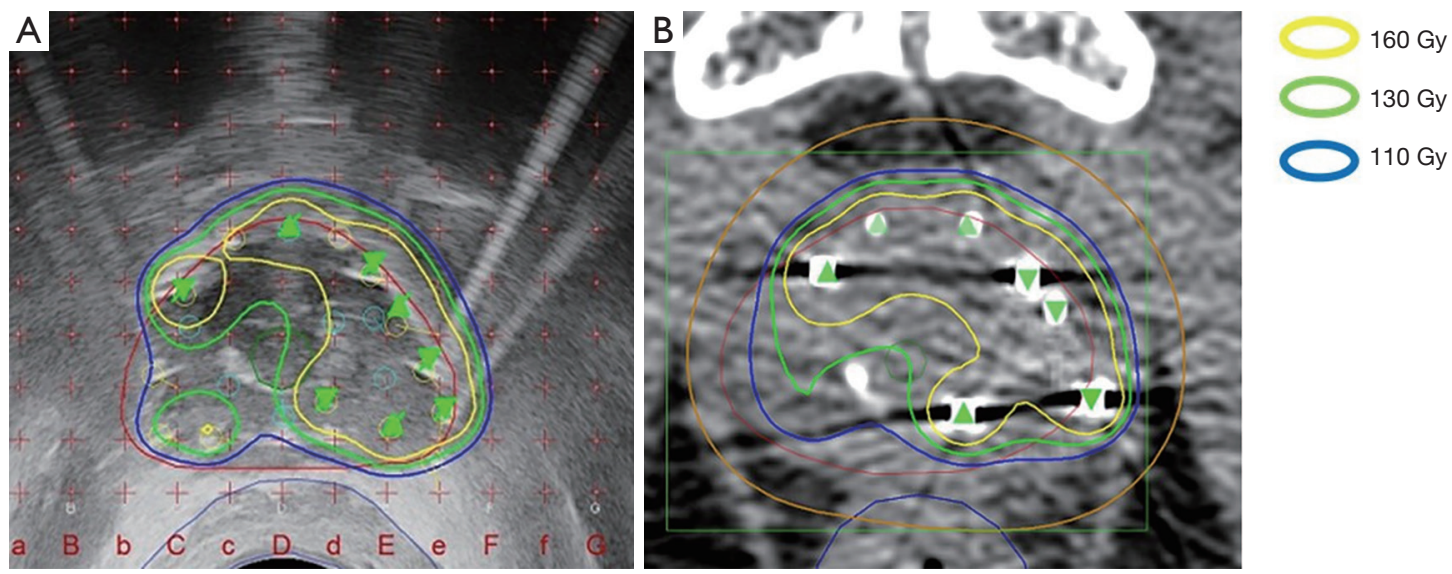

Figure 4 Dose cloud at intraoperative and at one month after salvage LDR BT by ${ }^{125}$ I seeds implantation in Case 2. (A) Axial dose cloud (160 Gy, yellow line) distribution during salvage LDR BT in Case $2 .{ }^{125}$ I seeds implantation covers over positive lesion per MRI and TGSM biopsy (the right lobe of the prostate) and keeps rectal dose to a minimum $(0 \mathrm{cc})$. The dose cloud at recurrent cancer-negative lesion proven by TGSM biopsy is designed to be limited to $130 \mathrm{~Gy}$ (green line) to $110 \mathrm{~Gy}$ (navy blue line) or lower. The red line indicates the prostate. (B) Axial dose cloud distribution one month after salvage LDR BT in Case 1. Intention of dose cloud design at the intraoperative phase is mostly achieved one month after salvage LDR BT. Red line indicates the prostate. LDR, low-dose-rate; BT, brachytherapy; MRI, magnetic resonance imaging; TGSM, template-guided saturation mapping.

recurrent prostate cancer after EBRT. We took biopsy samples from the MRI-based recurrent lesion in addition to the MRI-negative lesion to help minimize the dose to the areas where no cancer is present. These steps are crucial to map the recurrent cancer lesion accurately.

We used the MRI data and TGSM biopsy data to confine the seed radiation dose of 160 Gy to the recurrent cancer-positive lesion and keep R100 to a minimum $(0 \mathrm{cc})$. The dose to the recurrent cancer-negative lesion proven by TGSM biopsy should be 110 to 130 Gy or lower.

\section{Discussion}

Despite the relatively long history of salvage LDR BT $(3,5,6)$, its use in treating prostate cancer recurrence after EBRT contains several unsolved issues. Contemporary ideal candidates for salvage LDR BT are those who initially present as low- or intermediate-risk (i.e., PSA $<10 \mathrm{ng} / \mathrm{mL}$, biopsy Gleason score 6, T1c or T2a tumor status) $(3,5,6)$. Recurrence of high-risk prostate cancer usually precludes a patient from salvage treatment. The 2019 National Comprehensive Cancer Network (NCCN) guidelines limit the indication of local salvage therapy to primary stage $\mathrm{T} 1$ and T2 (7). Data are lacking on the outcomes of LDR BT in patients with high-risk prostate cancer recurrence. We also have to remind that the second round of radiotherapy for salvage LDR BT carries of risk of severe adverse effects, including high-grade GU or GI toxicities.

The follow-up periods for most studies have been too short to judge oncological outcomes (3-6,8-10). Burri et al. reported the most extended median follow-up for salvage LDR BT of 86 months. They found biochemical failurefree survival (BFFS) at ten years was $54 \%$ (11). Among the literatures of salvage LDR BT after EBRT failure, the report by Rose JN et al concluded that patient selection should focus on up to favorable intermediate risk cases while their data included several high-risk patients (10). In the same way, the radiation oncology group (RTOG) undertook a prospective Phase II trial to see the efficacy and toxicity of salvage LDRBT from 2007 to 2014 (RTOG 0526) (12): Eligible patients were limited to initial low to unfavorable intermediate risk prostate cancer (T1-T2c, Gleason score $\leq 7$, PSA <20).

Despite of the current trend of salvage LDR BT, our patient cases seem to indicate that salvage LDR BT can be a viable treatment option even for high-risk prostate cancer recurrence after EBRT.

We usually conduct ${ }^{125}$ I seed LDR BT with a minimal dose covering $90 \%$ of the prostate (D90) $>190$ Gy and the percent of the prostate receiving $100 \%$ of the prescribed dose (V100) >99\% (13). We showed excellent clinical outcomes with ${ }^{125} \mathrm{I}$ LDR-based radiotherapy in intermediate-risk patients, including a significant number of 
unfavorable intermediate-risk (UIR) cases with a BFFS rate of $99.1 \%$ at 7 years (13). ${ }^{125}$ I LDR alone with a biologically equivalent dose (BED) of $200 \mathrm{~Gy}$ is an optimal treatment for intermediate-risk prostate cancer patients, including UIR cases (13). Technical detail of the high dose and high quality LDR BT has been reported separately (14).

We also showed good clinical outcomes by escalating the dose for high-risk and very high-risk prostate cancer patients in our report of five pelvic lymph node metastasis cases with a BFFS rate of $95.2 \%$ at five years by LDR combined with EBRT BED >220 Gy (15).

Comparing our primary LDR BT (13). or LDR in combination with EBRT (15) with the present case data, the D90 and V100 for the whole prostate of the present salvage LDR BT cases are low (Table 3). This is because the present salvage LDR BT principles confine the high-dose cloud to the recurrent cancer-positive lesion and keep the radiation dose to the cancer-negative lesion to a minimum. Therefore, D90 or V100 is not an appropriate indicator for optimal salvage LDR BT. Currently, no consensus exists regarding the method or radiation dose for salvage LDR-BT (3).

Interestingly, in agreement with this author's opinion, RTOG-0526 toxicity data showed higher V100 predicted late Grade 3 adverse event in salvage LDR-BT after EBRT failure (12).

Prescription doses for salvage LDR BT range from $120-145$ Gy for ${ }^{125}$ I seed implantation and $90-135$ Gy for ${ }^{103} \mathrm{Pd}$ (3). Furthermore, there has been no consensus whether salvage LDR BT should be conducted on whole $(10,16)$ or partial glands $(9)$. Whole gland salvage with a higher dose did not result in good cancer control; instead, whole gland salvage resulted in severe toxicity $(3,10)$.

Chang et al. were the first to report on partial gland salvage LDR BT (9). They treated five initially low- or intermediate-risk patients who experienced local failure after EBRT. Their implantation method treated lobes unilaterally (right or left) with a prescription dose of $110 \mathrm{~Gy}$. The median follow-up after salvage LDR BT was 41 months. All five patients showed low GU and GI toxicities with a median latest PSA of $0.4 \mathrm{ng} / \mathrm{mL}$. Our salvage LDR BT method differed from Chang et al.'s, and both of our patients have stayed healthy without biochemical failure and bowel and urinary troubles as of their seven-year follow-up.

\section{Conclusions}

Salvage LDR BT is a viable treatment option for patients with high-risk prostate cancer recurrence. D90 or V100 is not an appropriate indicator for optimal salvage LDR BT in these difficult cases. In these problematic cases, the key to salvage LDR BT is confining the high-dose cloud to the recurrent cancer-positive lesion and keeping radiation doses to the cancer-negative lesion to a minimum.

\section{Acknowledgments}

The author thanks Dr. Naoaki Kohno, Department of Radiology, Shiga University of Medical Science for supporting dosimetric analysis.

Funding: None.

\section{Footnote}

Reporting Checklist: The author has completed the CARE reporting checklist. Available at https://dx.doi. org/10.21037/tro-21-12

Conflicts of Interest: The author has completed the ICMJE uniform disclosure form (available at https://dx.doi. org/10.21037/tro-21-12). KO was associated with the Department of Brachytherapy for Prostate Cancer endowed by Nihon Medi-Physics Co., Ltd. from 2015-2019. The present affiliation of Keisei Okamoto is Department of Urology, Uji Hospital, Kyoto, Japan.

Ethical Statement: The author is accountable for all aspects of the work in ensuring that questions related to the accuracy or integrity of any part of the work are appropriately investigated and resolved. All procedures performed in studies involving human participants were in accordance with the ethical standards of the institutional and/or national research committee(s) and with the Helsinki Declaration (as revised in 2013). Written informed consent was obtained from the patients for publication of this case report and accompanying images. A copy of the written consent is available for review by the editorial office of this journal.

Open Access Statement: This is an Open Access article distributed in accordance with the Creative Commons Attribution-NonCommercial-NoDerivs 4.0 International License (CC BY-NC-ND 4.0), which permits the noncommercial replication and distribution of the article with the strict proviso that no changes or edits are made and the original work is properly cited (including links to both the formal publication through the relevant DOI and the license). 
See: https://creativecommons.org/licenses/by-nc-nd/4.0/.

\section{References}

1. James LJ, Wong G, Craig JC, et al. Men's perspectives of prostate cancer screening: A systematic review of qualitative studies. PLoS One 2017;12:e0188258.

2. Nguyen PL, Gu X, Lipsitz SR, et al. Cost implications of the rapid adoption of newer technologies for treating prostate cancer. J Clin Oncol 2011;29:1517-24.

3. Tisseverasinghe SA, Crook JM. The role of salvage brachytherapy for local relapse after external beam radiotherapy for prostate cancer. Transl Androl Urol 2018;7:414-35.

4. Miyake M, Tanaka N, Asakawa I, et al. Oncological outcome, complications, lower urinary tract symptoms, and health-related quality of life after low-dose-rate salvage brachytherapy for recurrent prostate cancer following primary radiotherapy: a report of 8 cases. J Contemp Brachytherapy 2017;9:364-72.

5. Nguyen PL, D'Amico AV, Lee AK, et al. Patient selection, cancer control, and complications after salvage local therapy for postradiation prostate-specific antigen failure: a systematic review of the literature. Cancer 2007;110:1417-28.

6. Allen GW, Howard AR, Jarrard DF, et al. Management of prostate cancer recurrences after radiation therapy-brachytherapy as a salvage option. Cancer 2007;110:1405-16.

7. 2019 National Comprehensive Cancer Network Guidelines. 2019; Available online: https://www2.tri-kobe. org/nccn/guideline/urological/english/prostate.pdf

8. Aaronson DS, Yamasaki I, Gottschalk A, et al. Salvage permanent perineal radioactive-seed implantation for treating recurrence of localized prostate adenocarcinoma

doi: $10.21037 /$ tro-21-12

Cite this article as: Okamoto K. Can patients with recurrent high-risk prostate cancer after external beam radiotherapy (EBRT) be salvaged by low dose rate (LDR) brachytherapy: case report describing technique and application. Ther Radiol Oncol 2021;5:16. after external beam radiotherapy. BJU Int 2009;104:600-4.

9. Chang L, Buyyounouski MK. Salvage low-dose-rate (125)I partial prostate brachytherapy after dose-escalated external beam radiotherapy. J Contemp Brachytherapy 2014;6:304-10.

10. Rose JN, Crook JM, Pickles T, et al. Salvage low-doserate permanent seed brachytherapy for locally recurrent prostate cancer: Association between dose and late toxicity. Brachytherapy 2015;14:342-9.

11. Burri RJ, Stone NN, Unger P, et al. Long-term outcome and toxicity of salvage brachytherapy for local failure after initial radiotherapy for prostate cancer. Int J Radiat Oncol Biol Phys 2010;77:1338-44.

12. Crook JM, Zhang P, Pisansky TM, et al. A Prospective Phase 2 Trial of Transperineal Ultrasound-Guided Brachytherapy for Locally Recurrent Prostate Cancer After External Beam Radiation Therapy (NRG Oncology/RTOG-0526). Int J Radiat Oncol Biol Phys 2019;103:335-43.

13. Okamoto K, Okuyama K, Kohno N, et al. Clinical outcomes of low-dose-rate brachytherapy based radiotherapy for intermediate risk prostate cancer. J Contemp Brachytherapy 2020;12:6-11.

14. Okamoto K. Ten-step method of high-dose LDR 125I brachytherapy for intermediate-risk prostate cancer. J Appl Clin Med Phys 2021;22:172-82.

15. Okamoto K, Wada A, Kohno N. High biologically effective dose radiation therapy using brachytherapy in combination with external beam radiotherapy for high-risk prostate cancer. J Contemp Brachytherapy 2017;9:1-6.

16. Sutani S, Yorozu A, Toya K, et al. Whole-gland salvage treatment for recurrent prostate cancer after initial definitive radiotherapy: A case series of $125 \mathrm{I}$ brachytherapy and robot-assisted radical prostatectomy. J Contemp Brachytherapy 2019;11:201-6. 